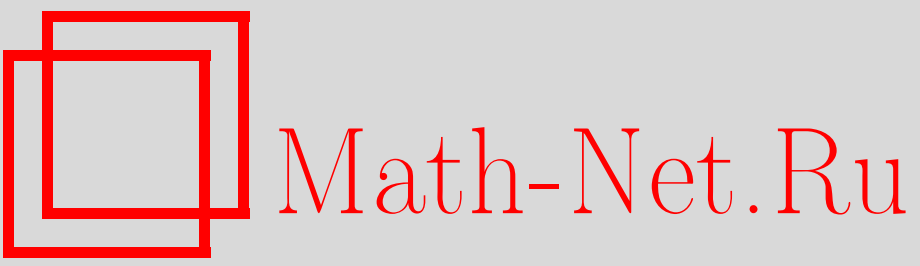

С. Ломбардо, Обратное спектральное преобразование для $q$-деформированного уравнения Вольтерра, ТМФ, 2002, том 133, номер 2, 259-269

DOI: https://doi.org/10.4213/tmf395

Использование Общероссийского математического портала Math-Net.Ru подразумевает, что вы прочитали и согласны с пользовательским соглашением

http://www.mathnet.ru/rus/agreement

Параметры загрузки:

IP: 3.85 .73 .92

26 апреля 2023 г., 08:44:06 


\section{ОБРАТНОЕ СПЕКТРАЛЬНОЕ ПРЕОБРАЗОВАНИЕ ДЛЯ q-ДЕФОРМИРОВАННОГО УРАВНЕНИЯ ВОЛЬТЕРРА}

Изучается $q$-деформация уравнения Вольтерра с помощью обратного спектрального преобразования. Вычислена зависимость $q$-деформированных спектральных данных от времени и явно построено односолитонное решение.

Ключевые слова: $q$-уравнения, $q$-деформации.

\section{1. ВВЕДЕНИЕ}

С уравнением Вольтерра, или моделью Вольтерра, обычно связывают дифференциально-разностные уравнения

$$
\frac{d c_{n}}{d t}=c_{n}\left(c_{n+1}-c_{n-1}\right), \quad n \in \mathbb{Z}, \quad c_{n}(t)>0
$$

Вольтерра использовал эту модель для изучения экологических проблем [1], однако она оказалась достаточно универсальной и имеет множество применений [2]. В последнее время интерес к различным версиям этой модели заметно возрос, в частности было обнаружено, что модель связана с уравнениями Лиувилля, и ее правильная гамильтонова интерпретация приводит к решеточной деформации алгебры Вирасоро [3]. Исследовался также квантовый вариант модели [4], а ее $q$-деформация, как выяснилось, естественно возникает как редукция подобия $q$-деформированного уравнения Пенлеве I [5]. Этот $q$-деформированный вариант уравнения Вольтерра является начальной точкой нашего исследования.

Насколько нам известно, $q$-деформация модели Вольтерра

$$
{ }_{q^{2}} D_{t} \bar{R}_{n}=q_{q^{2}}^{2} T_{t}\left(\bar{R}_{n} \bar{R}_{n+1}\right)-\bar{R}_{n} \bar{R}_{n-1}, \quad n \in \mathbb{Z},
$$

впервые появляется в статье Нийхоффа [5], посвященной установлению связи между $q$-деформацией дискретного уравнения Пенлеве I и $q$-деформацией ортогональных многочленов. Здесь $q^{a} D_{t}$ - оператор $q$-дифференцирования

$$
q^{a} D_{t} f(t) \equiv \frac{f\left(t q^{a}\right)-f(t)}{\left(q^{a}-1\right) t}=\frac{q^{a} T_{t} f(t)-f(t)}{\left(q^{a}-1\right) t}, \quad a \in \mathbb{Z},
$$

${ }^{*}$ Department of Applied Mathematics, University of Leeds, Leeds, UK.

E-mail: sara@maths.leeds.ac.uk 
$q^{a} T_{t}$ - оператор $q$-дилатации:

$$
q^{a} T_{t} f(t) \equiv f\left(t q^{a}\right), \quad a \in \mathbb{Z}
$$

В действительности мы будем изучать систему уравнений

$$
\begin{gathered}
q^{2} D_{t} R_{n}=Q_{n}-Q_{n+1} \\
\left({ }_{q^{2}} T_{t} R_{n}\right) Q_{n-1}=Q_{n} R_{n-2}
\end{gathered}
$$

которая сводится к (2) в предположении, что $\bar{R}_{n}=R_{n}+\left(1-q^{2}\right) t Q_{n+1}$.

Уравнения (5), (6) можно представить как условия совместности для системы линейных уравнений

$$
\begin{gathered}
\hat{\psi}_{n+1}+R_{n} \hat{\psi}_{n-1}=\lambda \hat{\psi}_{n} \\
{ }_{q^{2}} D_{t} \hat{\psi}_{n}=Q_{n} \hat{\psi}_{n-2}
\end{gathered}
$$

где уравнение (7) тесно связано со стандартной вспомогательной линейной задачей для модели Вольтерра (см. раздел 3), решенной много лет назад (см., например, [2]), тог да как уравнение (8) является $q$-дифференциальным уравнением; оно определяет временну́ю зависимость спектральных данных.

Целью данной работы являются нахождение временно́й зависимости спектральных данных и явное построение односолитонного решения системы (7), (8). Мы покажем, что решение имеет некоторые солитоноподобные черты, хотя оно и движется с непостоянной скоростью. При $q \rightarrow 1$ решение стремится к стандартному односолитонному решению уравнения (1).

Прежде чем двигаться дальше, обсудим очень важное свойство уравнений такого типа, характеризующее $q$-уравнения в целом. Несмотря на сходство $q$-производной и частной производной по времени (в действительности по любой переменной), их свойства достаточно различны. Действительно, в то время как из равенства $\partial_{t} f(t, \cdot)=0$ немедленно следует, что $f(t, \cdot)$ не зависит от времени $t$, имеется бесконечное множество зависящих от времени функций, которые принадлежат ядру оператора ${ }_{q} D_{t}$, т.е.

$$
\operatorname{Ker}\left({ }_{q} D_{t}\right) \equiv\left\{f(t, \cdot):{ }_{q} D_{t} f(t, \cdot)=0\right\}
$$

Например, любая функция $f(\ln t / \ln q)$, где $f(y)=f(y+1)$, принадлежит $\operatorname{Ker}\left({ }_{q} D_{t}\right)$. Поэтому необходимо обобщить задачу Коши так, чтобы обеспечить существование и единственность решения в этом случае. Для решения такой задачи мы ограничимся классом функций, мероморфных в окрестности $t=0$. Следующая лемма позволяет сформулировать задачу Коши для $q$-уравнений с обычными и частными производными.

ЛЕмма. Пусть $g(t)$-мероморфная функиия $t$ в открытой окрестности $t=0$. Пусть также $g(t) \in \operatorname{Ker}\left({ }_{q} D_{t}\right)$, m.e. ${ }_{q} D_{t} g(t)=0$. Тогда $g(t)-$ константа, т.е. не зависит от $t$. 
ДоКАЗАТЕЛЬСТво. Если $g(t)$ мероморфна вблизи $t=0$, ее можно представить в виде сходящегося ряда Лорана

$$
g(t)=\sum_{k=-n}^{+\infty} g_{k} t^{k}
$$

Тогда из ${ }_{q} D_{t} g(t)=0$ следует, что

$$
{ }_{q} D_{t} g(t)=\sum_{k=-n}^{+\infty} g_{k} t^{k-1} \frac{q^{k}-1}{q-1}=0
$$

откуда

$$
g_{k} t^{k-1} \frac{q^{k}-1}{q-1}=0,
$$

т.е. $g_{k}=0$ для любого $k \neq 0$. Следовательно,

$$
g(t)=g_{0}=\text { const }
$$

В дальнейшем мы будем предполагать, что все функции являются мероморфными в окрестности $t=0$.

\section{2. ПРЕДСТАВЛЕНИЕ ЛАКСА}

Проведем подробное дифференцирование $q$-уравнений Вольтерра (2), а также уравнений (5), (6). Сначала возьмем $q$-временну́ю производную уравнения $(7)$ :

$$
{ }_{q^{2}} D_{t} \hat{\psi}_{n+1}+_{q^{2}} D_{t}\left(R_{n} \hat{\psi}_{n-1}\right)=\lambda_{q^{2}} D_{t} \hat{\psi}_{n}
$$

Предполагая, что $q^{2} D_{t} \lambda=0$, и вспоминая $q$-модифицированное правило Лейбница

$$
q^{\alpha} D_{t}(f \cdot g)=\left({ }_{q^{\alpha}} D_{t} f\right) g(t)+f\left(q^{\alpha} t\right)\left({ }_{q^{\alpha}} D_{t} g\right)=\left({ }_{q^{\alpha}} D_{t} f\right) g\left(q^{\alpha} t\right)+f(t)\left({ }_{q^{\alpha}} D_{t} g\right),
$$

получим

$$
Q_{n+1} \hat{\psi}_{n-1}+{ }_{q^{2}} D_{t} R_{n} \hat{\psi}_{n-1}+R_{n}\left(q^{2} t\right) Q_{n-1} \hat{\psi}_{n-3}=\lambda Q_{n} \hat{\psi}_{n-2} \text {. }
$$

Используя теперь уравнение (7), разрешенное относительно $\hat{\psi}_{n-2} \quad\left(\lambda \hat{\psi}_{n-2}=\hat{\psi}_{n-1}+\right.$ $R_{n-2} \hat{\psi}_{n-3}$ ), мы можем переписать последнее выражение как

$$
\left(Q_{n+1}+{ }_{q^{2}} D_{t} R_{n}-Q_{n}\right) \hat{\psi}_{n-1}+\left(R_{n}\left(q^{2} t\right) Q_{n-1}-Q_{n} R_{n-2}\right) \hat{\psi}_{n-3}=0
$$

Полагая коэффициенты при членах $\hat{\psi}_{n-1}$ и $\hat{\psi}_{n-3}$ равными нулю, окончательно получаем соответственно уравнения (5) и (6). Из уравнения (5) следует, что

$$
{ }_{q^{2}} T_{t} R_{n}=R_{n}+\left(q^{2}-1\right) t\left(Q_{n}-Q_{n+1}\right) .
$$


Уравнение (6) можно теперь переписать в виде

$$
R_{n} Q_{n-1}+\left(q^{2}-1\right) t\left(Q_{n}-Q_{n+1}\right) Q_{n-1}=Q_{n} R_{n-2}
$$

и, перегруппировав члены, получить

$$
\frac{Q_{n}}{Q_{n-1}}=\frac{\bar{R}_{n}}{\bar{R}_{n-2}}
$$

где

$$
\bar{R}_{n}=R_{n}+\left(1-q^{2}\right) t Q_{n+1}
$$

Из соотношения (19) следует, что $Q_{n}$ можно представить в виде

$$
Q_{n}=-c \bar{R}_{n} \bar{R}_{n-1}
$$

где множитель $c$ не зависит от $n$, однако может зависеть от времени. Тогда из (5) получаем уравнение

$$
{ }_{q^{2}} D_{t} \bar{R}_{n}=q_{q^{2}}^{2} T_{t}\left(c(t) \bar{R}_{n} \bar{R}_{n+1}\right)-c(t) \bar{R}_{n} \bar{R}_{n-1}
$$

Выбор $c=$ const ( $c=1$ в работе [5]) приводит нас к уравнению (2), однако в этом случае $Q_{n}$ и $R_{n}$ имеют зависящие от времени асимптотики при $|n| \rightarrow \infty$.

Мы будем исследовать задачу, в которой $R_{n}$ и $Q_{n}$ имеют постоянные и не зависяшие от времени асимптотики. Поэтому выберем $Q_{n} \rightarrow-1, R_{n} \rightarrow 1$ при $|n| \rightarrow \infty$; отсюда сразу же следует, что

$$
c(t)=\left[1-\left(1-q^{2}\right) t\right]^{-2}
$$

\section{3. ПРЯМОЕ И ОБРАТНОЕ ПРЕОБРАЗОВАНИЯ РАССЕЯНИЯ}

Предположим, что все $R_{n}$ положительны, $R_{n} \rightarrow 1$ при $|n| \rightarrow \infty$ и бесконечное произведение

$$
\Delta_{\infty}=\prod_{n=-\infty}^{+\infty} R_{n}<\infty
$$

сходится. Оказывается, что если функции $R_{n}$ удовлетворяют системе $(5),(6)$, то $\Delta_{\infty}$ не зависит от времени; поэтому, если исходно она была ограничена, то остается ограниченной в любой момент времени $t$. Действительно, имеет место следующее

ПрЕДЛОЖЕНИЕ. Предположим, что $\Delta_{\infty}=\prod_{-\infty}^{+\infty} R_{k}-$ аналитическая функция в окрестности $t=0$. Тогда $\Delta_{\infty}$ постоянна. 
ДОкАЗАТЕЛЬСТво. Если вспомнить лемму, то оказьвается, что требуется лишь доказать $q^{2} D_{t} \Delta_{\infty}=0$, т.е. ${ }_{q^{2}} T_{t} \Delta_{\infty}=\Delta_{\infty}$. Имеем

$$
{ }_{q^{2}} T_{t} \Delta_{\infty}={ }_{q^{2}} T_{t}\left(\prod_{k=-\infty}^{\infty} R_{k}\right)=\prod_{k=-\infty}^{\infty}{ }_{q^{2}} T_{t} R_{k} .
$$

Теперь из уравнения (6) получаем равенство $q^{2} T_{t} R_{k}=Q_{k} R_{k-2} / Q_{k-1}$, подстановка которого в предыдушее соотношение дает искомый результат.

Простое калибровочное преобразование

$$
\psi_{n}=\sqrt{\Delta_{n}} \hat{\psi}_{n}, \quad \Delta_{n}=\prod_{k=n+1}^{\infty} R_{k},
$$

отображает задачу (7) в соответствуюшую задачу

$$
\sqrt{R_{n+1}} \psi_{n+1}+\sqrt{R_{n}} \psi_{n-1}=\lambda \psi_{n}
$$

для самосопряженного оператора

$$
L_{n m}=\sqrt{R_{n}} \delta_{n, m+1}+\sqrt{R_{m}} \delta_{n+1, m} .
$$

Связанные с этим оператором прямое и обратное преобразования рассеяния подробно изучены (см., например, [2]). Напомним кратко основные факты и определения, которые потребуются для нашего исследования.

Задача на собственные значения (27) имеет дважды вырожденный непрерывный спектр, заполняюший интервал $I=\{\lambda ;-2 \leqslant \lambda \leqslant 2\}$; соответствуюшие собственные функции ограничены для всех $n$. В общем случае задача (27) также может иметь вещественные дискретные собственные значения. Мы предполагаем, что их конечное число, т.е. что $J=\left\{\lambda_{1}, \ldots, \lambda_{N} ;\left|\lambda_{k}\right|>2\right\}$. Об этом множестве обычно говорят как о дискретном спектре, а соответствуюшие собственные функции являются квадратично-интегрируемыми.

Если $\lambda \in I$, то асимптотика $\psi_{n}$ при $|n| \rightarrow \infty$ имеет вид

$$
\psi_{n}=c_{1} z^{-n}+c_{2} z^{n}+o(1), \quad|z|=1, \quad \lambda=z+z^{-1} .
$$

Можно ввести функиии Йоста, т.е. специальные решения уравнения (27), определяемые асимптотическим поведением

$$
\psi_{n}=z^{n}+o(1) \text { при } n \rightarrow+\infty ; \quad \phi_{n}=z^{-n}+o(1) \text { при } n \rightarrow-\infty .
$$

Функции $\psi_{n}$ и $\phi_{n}$ аналитичны внутри единичной окружности в комплексной $z$-плоскости. Вторая пара специальных решений $\tilde{\psi}_{n}$ и $\tilde{\phi}_{n}$ определяется асимптотическим поведением

$$
\tilde{\psi}_{n}=z^{-n}+o(1) \text { при } n \rightarrow+\infty ; \quad \tilde{\phi}_{n}=z^{n}+o(1) \text { при } n \rightarrow-\infty .
$$


Они аналитичны вне единичной окружности.

Заметим, что эти функции, являясь решениями вешественнозначного уравнения с асимптотическими формами, задаваемыми вешественнозначными функциями $z$, удовлетворяют соотношениям $\bar{\psi}_{n}(z)=\psi_{n}(\bar{z}), \bar{\phi}_{n}(z)=\phi_{n}(\bar{z})$ и т.д. Очевидно, что на единичной окружности

$$
\tilde{\psi}_{n}(z)=\bar{\psi}_{n}(z), \quad \tilde{\phi}_{n}(z)=\bar{\phi}_{n}(z), \quad|z|=1 .
$$

Продолжая эти соотношения на комплексную $z$-плоскость, получаем

$$
\tilde{\psi}_{n}(z)=\bar{\psi}_{n}\left(\bar{z}^{-1}\right)=\psi_{n}\left(z^{-1}\right), \quad \phi_{n}\left(z^{-1}\right)=\tilde{\phi}_{n}(z)=\bar{\phi}_{n}\left(\bar{z}^{-1}\right) .
$$

На $z$-плоскости спектр является непрерьвным на единичной окружности $\left(\lambda=z+z^{-1}\right)$. При $|z|=1$ решения $\psi_{n}$ и $\bar{\psi}_{n}$ линейно независимы, а поскольку уравнение (27) имеет не более двух линейно независимых решений, то любое его решение должно быть линейной комбинацией $\psi_{n}$ и $\bar{\psi}_{n}$. В частности,

$$
\begin{gathered}
\phi_{n}=\alpha(z) \bar{\psi}_{n}+\beta(z) \psi_{n}, \quad|z|=1, \\
|\alpha(z)|^{2}-|\beta(z)|^{2}=1, \quad|z|=1 .
\end{gathered}
$$

Точки $z_{k}:|z|<1, k=1, \ldots, N$, в которых $\alpha(z)=0$, взаимно однозначно соответствуют собственным значениям дискретного спектра оператора $L: \lambda_{k}=z_{k}+z_{k}^{-1}$. В этих точках

$$
\phi_{n}\left(z_{k}\right)=b_{k} \psi_{n}\left(z_{k}\right)
$$

т.е. $\phi_{n}\left(z_{k}\right)$ являются соответствующими собственными функциями.

Назовем множество

$$
\mathcal{S}(n ; z)=\left\{z_{k}, b_{k}, r(z) \equiv \frac{\beta(z)}{\alpha(z)} ; k=1,2, \ldots, N\right\}
$$

спектральным преобразованием, а его элементы - данными рассеяния или спектральными данными для оператора $L$. Заметим, что для каждого $n$

$$
\psi_{n}(-z)=(-1)^{n} \psi_{n}(z), \quad \phi_{n}(-z)=(-1)^{n} \phi_{n}(z),
$$

так что

$$
\alpha(-z)=\alpha(z), \quad \beta(-z)=\beta(z), \quad|z|=1 .
$$

Таким образом, в терминах спектральных данных оператор $L$ выбирается согласно условию $r(-z)=r(z),|z|=1$. Более того, точки $z_{k}$ образуют множество, симметричное относительно $z=0$, и в симметричных точках этого множества значения $b_{k}$ одни и те же.

Имеется взаимно однозначное отображение между спектральными данными и последовательностью $R_{n}$; задача нахождения данных рассеяния для заданной последовательности $\left\{R_{n}\right\}$ называется прямым спектральныцм преобразованием. Решение обратной 
спектральной задачи, т.е. восстановление $R_{n}$ по заданному набору $\mathcal{S}(n, t ; z)$, подробно описано в работе [2].

Обратную спектральную задачу можно явно решить для безотражательных потенциалов, когда $r(z)$ тождественно равно нулю. Введем обозначение

$$
\phi_{n}(z)=z^{-n} \theta_{n}(z) \sqrt{\Delta_{n}}, \quad \tilde{\psi}_{n}(z)=z^{-n} \tilde{\chi}_{n}(z) \sqrt{\Delta_{n}}, \quad \psi_{n}(z)=z^{n} \chi_{n}(z) \sqrt{\Delta_{n}} .
$$

В силу уравнений $(33)$ имеем

$$
\tilde{\chi}_{n}(z)=\chi_{n}\left(z^{-1}\right) .
$$

Функции $\theta_{n}(z)$ и $\tilde{\chi}_{n}(z)$ аналитичны соответственно внутри и вне единичной окружности; $\tilde{\chi}_{n}(z)$ удовлетворяет соотношению

$$
z \tilde{\chi}_{n-1}+z^{-1} R_{n+1} \tilde{\chi}_{n+1}=\left(z+z^{-1}\right) \tilde{\chi}_{n}
$$

(такое же уравнение имеет место для $\left.\theta_{n}(z)\right)$. Из свойства аналитичности и уравнения (41) следует, что

$$
R_{n}=\frac{\tilde{\chi}_{n-1}(0)}{\tilde{\chi}_{n}(0)}
$$

Используя теперь уравнения (6), получаем

$$
Q_{n}=-\gamma(t) \frac{\tilde{\chi}_{n-2}(0)}{q^{2} T_{t} \tilde{\chi}_{n}(0)},
$$

где множитель $\gamma(t)$ можно определить из асимптотического поведения $\tilde{\chi}_{n}$ и $Q_{n}$; в нашем случае он оказывается равным единице. Тогда решение имеет вид

$$
R_{n}=\frac{\tilde{\chi}_{n-1}(0)}{\tilde{\chi}_{n}(0)}, \quad Q_{n}=-\frac{\tilde{\chi}_{n-2}(0)}{q^{2} T_{t} \tilde{\chi}_{n}(0)},
$$

где $\tilde{\chi}_{n}$ получается из решения обратной задачи.

В случае безотражательных потенциалов решение определяется характеристиками только дискретных спектров; в общем случае $\alpha(z)$ имеет $N$ нулей и записывается как

$$
\alpha(z)=\operatorname{sgn}\left(\prod_{k=1}^{N} z_{k}\right) \prod_{k=1}^{N} \frac{z-z_{k}}{z z_{k}-1} .
$$

В этом случае функция $\tilde{\chi}_{n}(z)$ является рациональной, и ее можно представить в виде

$$
\tilde{\chi}_{n}(z)=1+\sum_{m=1}^{N} \frac{z_{m}^{2 n} b_{m} \tilde{\chi}_{n}\left(z_{m}^{-1}\right)}{\alpha^{\prime}\left(z_{m}\right)\left(z-z_{m}\right)},
$$

где коэффициенты $\tilde{\chi}_{n}\left(z_{k}^{-1}\right), k=1, \ldots, N$, являются решениями замкнутой системы линейных алгебраических уравнений

$$
\tilde{\chi}_{n}\left(z_{k}^{-1}\right)=1+\sum_{m=1}^{N} \frac{z_{m}^{2 n} b_{m} \tilde{\chi}_{n}\left(z_{m}^{-1}\right)}{\alpha^{\prime}\left(z_{m}\right)\left(z_{k}^{-1}-z_{m}\right)} .
$$


Подробности этого вывода можно найти в работе [2].

В следуюшем разделе мы найдем временну́ю зависимость спектральных данных. В частности, мы покажем, что если $R_{n}$ и $Q_{n}$ удовлетворяют $q$-уравнению Вольтерра, то $\left\{z_{k}\right\}$ и $\alpha(z)$ не зависят от времени, тогда как $r(z)$ и $b_{k}$ являются явными функциями времени.

В простейшем случае (односолитонное решение) $\alpha(z)$ имеет два нуля: $z_{1}=z_{0}, z_{2}=$ $-z_{0},\left|z_{0}\right|<1$, и система алгебраических уравнений (48) состоит только из одного уравнения; его решение имеет вид

$$
\tilde{\chi}_{n}\left(z_{1}^{-1}\right)=\tilde{\chi}_{n}\left(z_{2}^{-1}\right)=A_{n}=\left(1+\frac{2 f(t) z_{0}^{2 n}}{\left(z_{0}^{-2}-z_{0}^{2}\right)}\right)^{-1}, \quad f(t)=-\frac{z_{0}}{\alpha^{\prime}\left(z_{0}\right)} b_{0}(t) .
$$

Отсюда

$$
\begin{gathered}
\tilde{\chi}_{n}(z ; t)=1-\frac{f(t) z_{0}^{2 n-1} A_{n}(t)}{\left(z-z_{0}\right)}+\frac{f(t) z_{0}^{2 n-1} A_{n}(t)}{\left(z+z_{0}\right)} \\
\left.\tilde{\chi}_{n}(z ; t)\right|_{z=0}=1+2 f(t) z_{0}^{2 n-2} A_{n}(t) .
\end{gathered}
$$

Тогда из соотношения (43) следует, что

$$
R_{n}=\frac{\tilde{\chi}_{n-1}(0)}{\tilde{\chi}_{n}(0)}=\frac{1+2 f(t) z_{0}^{2 n-4} A_{n-1}(t)}{1+2 f(t) z_{0}^{2 n-2} A_{n}(t)} .
$$

Аналогично из (44) следует, что

$$
Q_{n}=-\frac{\tilde{\chi}_{n-2}(0)}{q^{2} T_{t} \tilde{\chi}_{n}(0)}=-\frac{1+2 f(t) z_{0}^{2 n-6} A_{n-2}(t)}{1+2 f\left(q^{2} t\right) z_{0}^{2 n-2} A_{n}\left(q^{2} t\right)} .
$$

В частности, для односолитонного решения

$$
\Delta_{\infty}=\frac{\tilde{\chi}-\infty(0)}{\tilde{\chi}_{+\infty}(0)}=z_{0}^{-4}
$$

\section{4. ВРЕМЕННАЯ ЭВОЛЮЦИЯ СПЕКТРАЛЬНЫХ ДАННЫХ}

Чтобы найти временну́ю эволюцию спектральных данных, необходимо принять во внимание уравнение (8), описываюшее $q$-дифференциальную временни́ю әволюиию. Заметим, что спектральные данные были определены для задачи (27), которая отличается от (7) калибровочным преобразованием (26). Используя это преобразование, можно отождествить спектральные задачи для операторов, соответствуюших (7) и (27). Таким образом получаем

$$
\hat{\phi}_{n}=\alpha(z) \overline{\hat{\psi}}_{n}+\beta(z) \hat{\psi}_{n}
$$

где функции $\hat{\phi}_{n}, \overline{\hat{\psi}}_{\text {и }} \hat{\psi}_{n}$ удовлетворяют уравнению (7) и имеют асимптотики

$$
\begin{aligned}
& \hat{\phi}_{n} \rightarrow z^{-n}\left(\Delta_{\infty}\right)^{-1 / 2} \text { при } n \rightarrow-\infty, \\
& \hat{\psi}_{n} \rightarrow z^{n}, \quad \overline{\hat{\psi}}_{n} \rightarrow z^{-n} \text { при } n \rightarrow \infty .
\end{aligned}
$$


Аналогично стандартному подходу [2], из совместности (7) и (8), асимптотического вида функций Йоста и сформулированного нами предложения следует, что

$$
{ }_{q^{2}} D_{t} \hat{\phi}_{n}-Q_{n} \hat{\phi}_{n-2}=z_{q^{2}}^{2} T_{t} \hat{\phi}_{n}
$$

Подставляя (55) в (58), находим уравнения для $\alpha(z)$ и $\beta(z)$ :

$$
\begin{aligned}
& q^{2} D_{t} \alpha=-z^{2} \alpha+z_{q^{2}}^{2} T_{t} \alpha \\
& q^{2} D_{t} \beta=-z^{-2} \beta+z^{2}{ }_{q^{2}} T_{t} \beta
\end{aligned}
$$

Из (59) следует, что $q^{2} D_{t} \alpha=0$, а потому с учетом леммы получаем, что $\alpha(z)$ постоянна по времени.

Теперь рассмотрим уравнение (60). Используя определение $q$-производной $(3)$, для $\beta(z, t)$ получаем уравнение

$$
{ }_{q^{2}} D_{t} \beta=\frac{1-z^{-4}}{z^{-2}+\left(1-q^{2}\right) t} \beta .
$$

Аналогично находим, что $b_{k}(z, t)$ удовлетворяет такому же уравнению

$$
{ }_{q^{2}} D_{t} b_{k}=\frac{1-z^{-4}}{z^{-2}+\left(1-q^{2}\right) t} b_{k} .
$$

В частности, легко проверить, что

$$
{ }_{q^{2}} D_{t}\left(|\alpha|^{2}-|\beta|^{2}\right)=0, \quad|z|=1
$$

Чтобы решить уравнения (61) и (62), перепишем их (например, уравнение для $\beta$ ) как

$$
{ }_{q^{2}} T_{t} \beta=\frac{1-\left(q^{2}-1\right) z^{-2} t}{1-\left(q^{2}-1\right) z^{2} t} \beta
$$

т.e.

$$
\beta\left(q^{2} t\right)=\frac{1-\left(q^{2}-1\right) z^{-2} t}{1-\left(q^{2}-1\right) z^{2} t} \beta(t) .
$$

После $k$ итераций уравнения (65) получим

$$
\beta\left(q^{2 k} t\right)=\prod_{j=0}^{k} \frac{1-\left(q^{2}-1\right) z^{-2} q^{2 j} t}{1-\left(q^{2}-1\right) z^{2} q^{2 j} t} \beta(t) .
$$

Отсюда, предполагая $|q|<1$, в пределе $k \rightarrow \infty$ имеем

$$
\beta(0)=\prod_{j=0}^{\infty} \frac{1-\left(q^{2}-1\right) z^{-2} q^{2 j} t}{1-\left(q^{2}-1\right) z^{2} q^{2 j} t} \beta(t),
$$


что дает

$$
\beta(t)=\frac{\left(\left(q^{2}-1\right) z^{2} t ; q^{2}\right)_{\infty}}{\left(\left(q^{2}-1\right) z^{-2} t ; q^{2}\right)_{\infty}} \beta(0),
$$

где мы использовали обозначения

$$
(a ; q)_{\infty} \equiv \prod_{k=0}^{\infty}\left(1-a q^{k}\right), \quad(a ; q)_{0}=1 ; \quad|q|<1, \quad a \in \mathbb{C} .
$$

Уравнение (68) можно переписать в терминах q-экспонент [6], которые определяются каK

$$
\begin{aligned}
e_{q}(\zeta) & \equiv \sum_{k=0}^{\infty} \frac{\zeta^{k}}{(q ; q)_{k}}=\frac{1}{(\zeta ; q)_{\infty}}, \quad|\zeta|<1, \\
E_{q}(\zeta) & \equiv \sum_{k=0}^{\infty} \frac{q^{k(k-1) / 2} \zeta^{k}}{(q ; q)_{k}}=(-\zeta ; q)_{\infty}, \quad \zeta \in \mathbb{C}
\end{aligned}
$$

$e_{q}(\zeta)$ и $E_{q}(\zeta)$ представляют собой наиболее элементарные $q$-гипергеометрические ряды и являются $q$-аналогами стандартных экспонент в соответствии с предельными формулами

$$
\lim _{q \rightarrow 1} E_{q}((1-q) \zeta)=e^{\zeta}=\lim _{q \rightarrow 1} e_{q}((1-q) \zeta) .
$$

Здесь предел должен рассматриваться почленно; иногда он обозначается как $\lim _{q \uparrow 1}$.

Используя эти обозначения, можно окончательно выразить решения уравнений (61), (62) в терминах әлементарных q-функиий, т.е.

$$
\begin{aligned}
\beta(t) & =e_{q^{2}}\left(\left(q^{2}-1\right) z^{-2} t\right) E_{q^{2}}\left(\left(1-q^{2}\right) z^{2} t\right) \beta(0), \\
b_{k}(t) & =e_{q^{2}}\left(\left(q^{2}-1\right) z_{k}^{-2} t\right) E_{q^{2}}\left(\left(1-q^{2}\right) z_{k}^{2} t\right) b_{k}(0) .
\end{aligned}
$$

В частности (с учетом (49)), получаем функцию

$$
f(t)=e_{q^{2}}\left(\left(q^{2}-1\right) z_{0}^{-2} t\right) E_{q^{2}}\left(\left(1-q^{2}\right) z_{0}^{2} t\right) f(0)
$$

которая, очевидно, аналитична в окрестности $t=0$.

Окончательно, подставляя (75) в (49), (52) и (44), получаем в явном виде односолитонное решение $q$-уравнения Вольтерра.

Отметим, что, используя (72), мы получаем

$$
\lim _{q^{2} \uparrow 1} \beta(t)=e^{\left(z^{2}-z^{-2}\right) t} \beta(0), \quad \lim _{q^{2} \uparrow 1} b_{k}(t)=e^{\left(z_{k}^{2}-z_{k}^{-2}\right) t} b_{k}(0),
$$

т.е. стандартную временную зависимость (см. [2]). Отсюда в пределе $q \rightarrow 1$ воспроизводится односолитонное решение уравнения (1).

Сравнивая $q$-деформированное решение с решением уравнения (1) (см., например, [2]), можно сделать вывод о том, что при фиксированном $t$ оба решения имеют одну и ту же форму (как функции $n$ ). Напротив, временные зависимости различны (см. рисунок): 

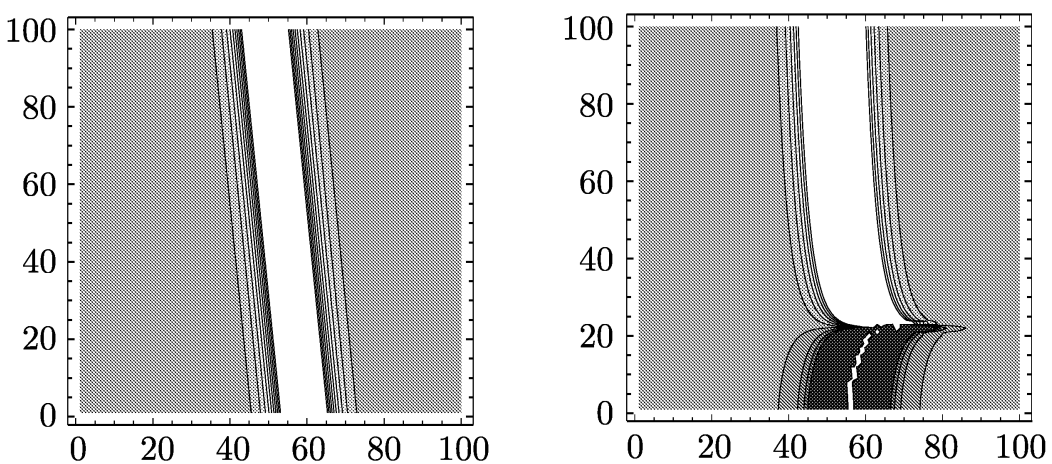

Представление функции $R_{n}$ изменением плотности изображения. Левая часть рисунка соответствует солитонному решению модели Вольтерра, а правая - солитонному решению $q$-деформированной модели Вольтерра (по осям отложены $t$ и $n$ ).

$q$-деформированное решение перемещается с непостоянной скоростью вследствие сингулярностей (полюсов) функции $e_{q^{2}}\left(e_{q}(\zeta)\right.$ является мероморфной функцией с простыми полюсами при $\left.\zeta=q^{-k}, k \in \mathbb{Z}\right)$.

Благодарности. Я благодарна А. В. Михайлову за то, что он привлек мое внимание к этой задаче, за полезные советы и поошрение к опубликованию данной работы, а также В. Кузнецову и $\Phi$. Нийхоффу за стимулируюшие обсуждения. Я признательна за гостеприимство сотрудникам Университета Лидса и Института математических наук Исаака Ньютона в Кембридже, где была выполнена часть данной работы в рамках программы "Интегрируемые системы".

\section{Список литературы}

[1] V. Volterra. Leçn sur la théorie mathématique de la lutte pour la vie. Paris: Guatier-Villars, 1931.

[2] В. Е. Захаров, С. В. Манаков, С. П. Новиков, Л. П. Питаевский. Теория солитонов: Метод обратной задачи. М.: Наука, 1980.

[3] L. D. Faddeev, L. A. Takhtajan. Liouville model on the lattice. In: Field Theory, Quantum Gravity and Strings. Lect. Notes Phys. V. 246. Eds. J. de Vega, N. Sánchez. Berlin: Springer, 1986. P. 66.

[4] A. Yu. Volkov. Phys. Lett. A. 1992. V. 167. P. 345-355; A. В. Антонов. ТМФ. 1997. Т. 113. № 3. C. 384-396; hep-th/9607031.

[5] F. W. Nijhoff. Lett. Math. Phys. 1994. V. 30. P. 327-336.

[6] T. H. Koornwinder. $q$-Special function, a tutorial. math.CA/9403216; Special function and $q$-commuting variables. $q$-alg/9608008. 